punto org

Collana diretta da Luigi Maria Sicca

24 
LUCA PARESCHI

\title{
CONTROCAMPO LETTERARIO
}

Strategie di intermediazione e accesso

all'industria editoriale

\author{
prefazione \\ Paola Dubini \\ postfazione \\ Gianluca Colombo
}

Editoriale Scientifica

Napoli 
Tutti i diritti riservati

(C) 2016 Editoriale Scientifica srl

Via San Biagio dei Librai, 39

80138 Napoli

www.editorialescientifica.com

info@editorialescientifica.com

ISBN 978-88-6342-935-0 


\section{Indice}

9 Prefazione di Paola Dubini

17 1. INTRODUZIONE

23 2. QUADRO DI RIFERIMENTO TEORICO

23 1. Il campo letterario

27 2. Habitus, capitale

$303 . \quad$ L'intermediazione

34 4. Incertezza estetica e intermediazione

37 5. Intermediazione nel campo editoriale

40 6. Altri campiculturalie intermediazione

49 3. STORIA E NUMERI DEL CAMPO LETTERARIO ITALIANO

49 7. Introduzione

49 8. Storia dell'editoria letteraria in Italia

60 9. Le fonti di dati sull'industria editoriale italiana

69 10. Misure sul capo editoriale italiano

7010.1 Produzione

$83 \quad 10.2$ Distribuzione

$91 \quad 10.3$ Il consumo

93 11. Conclusione

95 4. Problema e Disegno Della RicerCA

95 12. La ricerca

100 13. Disegno della ricerca

$101 \quad 13.1$ La prima fase: case editrici

$102 \quad 13.2$ La seconda fase: agenti del campo letterario

$120 \quad 13.3$ Analisi delle interviste 
14. Appendice

14.1 Domande per editor, editori, direttori editoriali o di collana

14.2 Domande per gli agenti letterari

14.3 Domande per gli altri agenti costituenti il campo letterario

5. IL CAMPO LETTERARIO ITALIANO

131

15. Introduzione

133

16. Una cittadella fortificata

138

138

17. Agenti e istituzione nel campo letterario

145

17.1 Case editrici

148

151

158

17.2 Aspiranti scrittori

17.3 Agenti letterari

17.4 Scrittori, intellettuali ed altri agenti del campo letterario

17.5 Editor e lettori

162

17.6 Scuole di scrittura

166

167

17.7 Manifestazioni per esordienti

17.8 Premi letterari

17.9 Riviste letterarie

173

173

6. I CRITERI DI SELEZIONE DEI MANOSCRITTI

18. Le caratteristiche dei manoscritti

18.1 Lingua, voce, stile

18.2 Storia, tema, struttura

186

18.3 "Qualcosa che possa passare a altri»; "leggo e non mi accorgo che è lavoro»

18.4 Focus, «manico»

18.5 Altre considerazioni

18.6 Le parole sono importanti

18.7 Non un elenco della spesa

19. Valore letterario e successo commerciale

19.1 Le parole per dirlo 
210 20. Cercare autori o manoscritti

212 21. La versione degli agenti letterari

220 22. La versione degli intermediari

229 23. I criteri di selezione: conclusioni provvisorie

234 24. Appendice: due esempi di schede di scheda di lettura

23424.1 Prima scheda di lettura: CCC, di Alessandro HHH

23724.2 Seconda scheda di lettura: Tommaso XXX, YYY ZZZ

241 7. Processi di AMMISSIONE AL CAMPO LETTERARIO

24125 . Il ruolo degli editor

248 26. Pubblicare esordienti

$248 \quad 26.1$ Il piacere della scoperta

25126.2 Economia dell'esordio

25526.3 Promuovere un esordiente

$258 \quad 26.4$ Tirature

26626.5 Età della casa editrice

267 27. Opere straniere

271 28. Accesso alle case editrici

27128.1 Case editrici grandi

$279 \quad 28.2$ Case editrici medie

28428.3 Case editrici piccole

291 29. Il ruolo degli agenti letterari

29829.1 Vendere romanzi agli editor

30029.2 Valutazioni di manoscritti a pagamento

303 30. Appendice: tre esempi di contratto di valutazione di manoscritti a pagamento

$303 \quad 30.1$ Marco Vigevani Agenzia Letteraria: servizio di lettura manoscritti

30530.2 Agenzia Letteraria Internazionale: Servizio di valutazione

$306 \quad 30.3$ Grandi E Associati: servizio Valutazione Inediti

309 8. INTERMEDIARI OCCASIONALI E SERIALI

309 31. Intermediari occasionali 
316 32. Intermediari seriali

316

319

32.1 Chi sono gli intermediari seriali

320

32.2 La ricompensa

328

32.3 Come mediano gli intermediari seriali

334

32.4 I motivi dell'intermediazione

340

32.5 La legittimazione come intermediario seriale

342 32.6 Gli stessi intermediari per libri diversi

344

32.7 Due traiettorie nel campo letterario

345

33. Tre esempi di esordio

348

353

33.1 Roberto Saviano, Gomorra

33.2 Paolo Giordano, La solitudine dei numeri primi

33.3 Silvia Avallone, Acciaio

357 9. Conclusione

373 Ringraziamenti

375 Postfazione di Gianluca Colombo

377 Bibliografia

$391 \quad$ Indice dei Nomi

401 Hanno scritto nella collana punto org 


\title{
Prefazione
}

\author{
Paola Dubini*
}

Il libro di Luca Pareschi affronta un tema rilevante per gli studiosi di management e per gli operatori, oltre a parlare del campo da cui sono partita nella mia ricerca personale e cui rimango profondamente attaccata e interessata. E quindi mi fa davvero piacere introdurlo e - con questa scusa - riflettere su alcune delle questioni che solleva.

Il libro parla della filiera editoriale libraria (in particolare quella letteraria, in particolare i suoi anelli a monte, in particolare rispetto alla identificazione, alla scelta e alla legittimazione degli autori esordienti) come un campo, à la Bourdieu. Questo taglio mi sembra una scelta felice: innanzitutto perché fa "sentire a casa" gli operatori, in particolare gli editori e chi in casa editrice lavora. E quindi invita ad una lettura ragionata di un autore che ha sul settore uno sguardo non comune, visto che proviene da una disciplina spesso lontana dalle frequentazioni abituali di chi lavora in una casa editrice letteraria. Le case editrici tendono a guardare più spesso "a monte" e meno spesso "a valle", nella riflessione sui modi più appropriati per sostenere la qualità del proprio lavoro, la propria reputazione, la propria economicità. E quindi il libro parla a chi lo legge per trarre spunto per il suo lavoro, mettendosi nelle condizioni di farsi ascoltare. Perché dice

* Professore associato di Economia Aziendale, Università Commerciale Luigi Bocconi, Milano. 
cose utili e interessanti, poiché parla del cuore del problema.

Il riferimento alla teoria di Bourdieu, e ci viene ben spiegato, è molto adatto per studiare l'oggetto di indagine di questo libro, perché Bourdieu guardava proprio a questi tipi di settori, e quindi per una volta non dobbiamo trasferire teorie e modelli manageriali pensati altrove ad oggetti di indagine un po' sfuggenti, ma possiamo "usare" teorie costruite attorno alla specificità di questi settori per capire che cosa la letteratura e la pratica manageriale hanno da imparare dalle organizzazioni che si occupano di cultura.

E infine, la teoria di Bourdieu ci costringe a guardare all'oggetto di indagine come ad un sistema interconnesso, in cui contano non solo i singoli attori, ma le regole e le relazioni che li legano; settori di cui si entra a far parte per cooptazione da una parte, per affinità dall'altra e in cui opportunismo, calcolo economico, volontà politica, intuizione, comunità di intendimenti, identità e senso di appartenenza si mescolano continuamente. Per uno studioso di management questo è un ambito in cui la teoria delle reti, la logica istituzionale e tutte le teorie che da queste hanno preso le mosse e con queste si sono confrontate sono strumenti teorici utilissimi per comprendere come si entra, si cresce, si viene legittimati, si diventa famosi, come si ha successo economico. I concetti di intermediario, broker e gatekeeper assumono all' interno di questo campo di analisi sfumature rilevanti in una prospettiva di theory building.

L'oggetto di analisi è l'interazione dell'insieme di attività e di attori che concorrono alla pubblicazione di un libro mai pubblicato prima, in altre parole come si entra nel campo dal punto di vista di chi ci è già dentro. Il campo letterario ci viene presentato come un "campo di forze e un campo di lotte", ma è allo stesso tempo un campo di mediazioni culturali ed economiche continuo: la strada che separa l'autore dal suo lettore è piena di mediatori che svolgono funzioni di scouting, di selezione, di inclusione, di trasmissione, di integrazione, di gatekeeping, di promozione, 
di advocacy, per convinzione, per interesse, per passione, per opportunismo, per mestiere. In buona parte a ciascun attore spetta un ruolo diverso, e infatti abbiamo nomi diversi per definire gli attori che compongono il campo e che mettono in atto le lotte e le forze di cui leggerete nel corso del libro: le scuole di scrittura, gli agenti, gli scout, gli editor, i critici, e poi i distributori, i librai, i giornalisti... Ma spesso tali attori giocano più ruoli, non uno solo. E inoltre questo sistema di forze e di lotte finalizzato a stabilire chi entra e chi sta fuori dal campo non è solo antagonistico: questi stessi attori non solo escludono, spesso includono, integrano, traducono nel senso di mettere in relazione, come quando un editore sceglie di inserire un titolo in una specifica collana, o di abbinare un autore ad uno specifico illustratore, quando un agente richiede che la copertina del libro per l'edizione straniera sia la stessa dell'edizione originale, o quando viceversa la scelta della copertina, della collana, dei titoli da tradurre di uno specifico autore da parte di un editore straniero contribuiscono ad un posizionamento diverso dell'autore sulla scena culturale di un dato paese. Insomma, è proprio vero che è il campo nel suo complesso a costruire il libro come prodotto culturale e ad attribuirgli un valore. E a consacrare, a rendere riconoscibile l'autore. E per quanto gli attori giochino ruoli specifici, le contaminazioni sono importanti: come in tutti i settori culturali, chi scrive libri è spesso un avido lettore; la biografia di Calvino (autore, scout, traduttore), ma anche quella di molte delle persone intervistate nel libro ci mostra come il processo di crescita professionale e di legittimazione individuale all'interno del campo passi anche dall'appartenenza ad una rete di relazioni attraverso l'assunzione di ruoli diversi; le lettere di Linder ai suoi autori e agli editori italiani e stranieri (così come le lettere degli autori ai loro editori) ci rivelano rapporti articolati di natura umana e commerciale; gli editori proteggono l'integrità dell'opera dell'autore, ma allo stesso tempo contribuiscono al loro posizionamento sui diversi mercati: si pensi alle operazioni commerciali di Adelphi con 
Schopenauer o degli editori di Guareschi in Italia e in Germania. Per me lettrice, l'immaginario che ha costruito Rodari è strettamente collegato alle illustrazioni di Munari; per le mie figlie, $\mathrm{i}$ personaggi amatissimi di Roald Dahl non sarebbero gli stessi senza il tratto di Quentin Blake.

Proprio perché l'insieme delle forze e dei ruoli e degli attori nel processo di selezione di un autore e di un titolo da pubblicare costituisce una matassa piuttosto intricata, è utile l'approccio del libro: dipanare i nodi partendo dalle pratiche per definire i ruoli di mediazione e permettere così uno studio dell'intermediazione fra l'autore e il suo lettore.

L'oggetto di analisi è l'intermediazione editoriale che porta alla pubblicazione degli autori esordienti, a partire dalla constatazione che quando Feltrinelli decise di pubblicare il Gattopardo, l'accessibilità di un editore per un autore poco noto era ragionevolmente alta, mentre ora la fortezza della casa editrice è protetta da una serie di interlocutori che lasciano percolare pochissimi nomi. Ed è interessante notare come la ricerca "di una storia che piace ben scritta" (perché questo emerge evidente come l'elemento comune della fatica degli attori coinvolti) di autore ignoto sia un processo collettivo, del campo appunto, ancora molto artigianale, pur in un settore che ha assunto caratteri industriali e fortemente concentrato. Una cosa emerge e fa riflettere: nella narrativa le prime edizioni rappresentano quasi il $70 \%$ dei titoli pubblicati in un anno. Il settore editoriale per rimanere vitale ha quindi bisogno di continue nuove immissioni, non solo di nuovi titoli di autori affermati, ma anche di genuine novità.

La capacità di alimentare la sostenibilità economica di una casa editrice deriva dalla possibilità di continuare a pubblicare nuovi titoli di autori in grado di affermarsi sul mercato: i titoli che hanno avuto successo in passato, i titoli di catalogo, meglio se long seller, coprono i costi fissi e proteggono dai giorni di pioggia, ma sono le novità che fanno il fatturato, che danno visibilità, che alimentano il sistema. Le case editrici continuano a proporre 
novità perché questa è la regola del gioco, sono i venture capitalist culturali, svolgono la funzione dei galleristi nel settore dell'arte contemporanea. E come nell'arte contemporanea i 200 galleristi che espongono ad Art Basel sono i gatekeeper di questo mercato, così gli editori, nel loro piccolo, fanno lo stesso. Per un autore, soprattutto letterario e soprattutto all'inizio della carriera, chi lo pubblica è molto importante, perché condiziona il suo successo commerciale e reputazionale. E ad un autore servono entrambi. È chiaro che come un venture capitalist costruisce la sua reputazione e la sua ricchezza quanto più è in grado di "fiutare" progetti imprenditoriali con potenziale quando sono piccoli e sconosciuti, così l'editore capace di scovare esordienti in gamba o lanciare autori sconosciuti vedrà crescere i suoi risultati su entrambi i fronti. E non stupisce che siano i piccoli editori quelli più attenti a scovare esordienti promettenti: la parte più interessante dei campi, quando vogliamo capire l'innovazione, viene dai bordi. E questo libro ci fa capire le mille porosità dei bordi del campo, note a chi ne fa parte, difficili da capire per chi ne sta fuori.

C'è un grande assente in questo libro (ma questa è una constatazione, non una critica) ed è l'autopubblicazione, considerata solo nella sua componente di editoria di vanità. In realtà, a me pare, l'autopubblicazione non è fatta solo di editoria di vanità. Il campo indagato in questo volume - così ben protetto dalla molteplicità delle relazioni, dalla permeabilità dei ruoli, dalle relazioni di influenza non necessariamente economiche - mantiene un connotato umano e artigianale messo in discussione dall'avvento delle piattaforme digitali, che hanno saputo costruire su una scala industriale incompatibile con le dinamiche e i tempi del settore editoriale librario un mercato dei titoli esordienti costruito "a rovescio" rispetto al campo, ossia a partire dal gradimento dei lettori. È un po' come dire che da una parte abbiamo il campo descritto in questo libro, in cui il sistema editoriale cerca fra gli esordienti "una storia ben scritta che piace" e le cerca un mercato e dall'altra un mercato che rimbalza gli esercizi dell'au- 
tore esordiente a proporre "una storia che piace possibilmente ben scritta" e poi la porge alla casa editrice perché la consacri. Forse per capire le relazioni pericolose fra questi due modi diversamente spietati per selezionare gli autori esordienti (perché che il processo sia draconianamente darwiniano lo si capisce benissimo, in entrambi i casi, anche se in modi molto diversi) occorre interrogare proprio gli autori. Speriamo quindi che questo libro abbia un sequel... 